\title{
Hamilton-Jacobi formalism for Podolsky's electromagnetic theory on the null-plane
}

Cite as: J. Math. Phys. 58, 082902 (2017); https://doi.org/10.1063/1.4999846

Submitted: 13 February 2017 . Accepted: 11 August 2017 . Published Online: 25 August 2017

M. C. Bertin (D), B. M. Pimentel, C. E. Valcárcel, and C. E. R. Zambrano
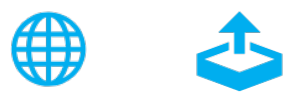

\section{ARTICLES YOU MAY BE INTERESTED IN}

Higher-derivative generalization of conformal mechanics

Journal of Mathematical Physics 58, 082903 (2017); https://

doi.org/10.1063/1.4986367

Lie and Noether point symmetries for a class of nonautonomous dynamical systems

Journal of Mathematical Physics 58, 082901 (2017); https:/ /

doi.org/10.1063/1.4998715

\section{Quantum mechanics of a photon}

Journal of Mathematical Physics 58, 082302 (2017); https://

doi.org/10.1063/1.4999847

\section{Where in the world is AIP Publishing?}

Find out where we are exhibiting next 


\title{
Hamilton-Jacobi formalism for Podolsky's electromagnetic theory on the null-plane
}

\author{
M. C. Bertin,, ,a) B. M. Pimentel, ${ }^{2, b)}$ C. E. Valcárcel, ${ }^{3, c)}$ \\ and G. E. R. Zambrano ${ }^{4, d)}$ \\ ${ }^{1}$ Instituto de Física, Universidade Federal da Bahia, Câmpus Universitário de Ondina, \\ 40210-340 Salvador, Brazil \\ ${ }^{2}$ Instituto de Física Teórica, UNESP-Sao Paulo State University, P. O. Box 70532-2, \\ 01156-970 São Paulo, Brazil \\ ${ }^{3}$ Centro de Matemática, Computação e Cognição, Universidade Federal do ABC, \\ 09210-170 Santo André, SP, Brazil \\ ${ }^{4}$ Departamento de Física, Universidad de Nariño, Calle 18 Carrera 50, San Juan de Pasto, \\ Nariño, Colombia
}

(Received 13 February 2017; accepted 11 August 2017; published online 25 August 2017)

\begin{abstract}
We develop the Hamilton-Jacobi formalism for Podolsky's electromagnetic theory on the null-plane. The main goal is to build the complete set of Hamiltonian generators of the system as well as to study the canonical and gauge transformations of the theory. Published by AIP Publishing. [http://dx.doi.org/10.1063/1.4999846]
\end{abstract}

\section{INTRODUCTION}

In this paper, we analyse the null-plane canonical structure of the generalised Podolsky's electrodynamics via the Hamilton-Jacobi (HJ) formalism for singular systems. Since we are dealing with three separate subjects, each one deserving proper treatment by itself, we separate the work into three main parts that correspond to our Secs. II-IV.

In Sec. II, the general Hamilton-Jacobi theory for singular and higher-order Lagrangian systems is presented, as it is the case of Podolsky's theory. The $\mathrm{HJ}$ formalism is well known from classical mechanics to be a road for the study of integrability of classical systems, but its fundamental role in dynamical systems was discovered only after the work of Carathéodory ${ }^{1}$ on variational principles and first-order partial differential equations (PDEs). Carathéodory built the HJ theory directly from Hamilton's principle, showing that it is actually the theory that relates first-order PDEs, first-order ordinary differential equations (ODEs), and Lagrangian variational problems. This unifying point of view is called the complete figure of the variational calculus and congregates all analytic, algebraic, and geometric pictures of mechanics. ${ }^{2}$

Considering singular systems, which are theories whose Lagrange function has singular Hessian matrices, the HJ theory does not exhibit the same fundamental problems seen when the purely Hamiltonian picture is considered. ${ }^{3,4}$ Carathéodory's complete figure is still valid, and a general treatment of constraints is naturally emergent. ${ }^{5}$ In the HJ theory, canonical constraints are seen as a set of Hamilton-Jacobi partial differential equations (HJPDEs), and the integrability of this set is achieved by an integrability theorem, leading to Frobenius' integrability conditions. ${ }^{6,7}$ Advantages of the $\mathrm{HJ}$ approach come from the fact that it provides a full theoretical stage to the canonical formulation of singular systems, rather than a consistency construction. One of the advantages is the absence of the so-called Dirac's conjecture. Also, gauge fixing is not required for equivalence between the canonical and Lagrangian descriptions at the classical level.

\footnotetext{
a)mbertin@ufba.br

b) pimentel@ift.unesp.br

c) carlos.valcarcel@ufabc.edu.br

d) gramos@udenar.edu.com
} 
The interest in higher derivative Lagrangian systems has been renewed from time to time because of some desired properties when describing quantum fields and gravity. Quadratic terms of the curvature and its contractions can be used in the Einstein-Hilbert action in order to solve the problem of renormalisation of the linearised gravitational field. ${ }^{8}$ Gravitational models with these terms exhibit interesting physical features, such as the existence of massive spin-two and massive scalar excitations. ${ }^{9}$ In 3D gravitational models,${ }^{10}$ higher-order terms have been used as laboratory to better understand the quantisation of the gravitational field and the general character of the holographic conjecture. ${ }^{11}$ Investigation of higher-order effective gauge theories ${ }^{12}$ and of the infrared sector of QCD shows good asymptotic behavior of the gluon propagator. ${ }^{13}$ In general, convergence of Feynman diagrams is improved with the presence of higher derivatives in the Lagrangian density.

Second and higher derivative Lagrangian systems were treated via the HJ formalism in Ref. 14. High-order theories were introduced by Ostrogradsky ${ }^{15}$ back in 1850, but comprehensive reviews of the Ostrogradsky method can be found in Refs. 16 and 17 along with classical mechanical examples. In Ref. 16, the authors also discuss the problem of instability of the Ostrogradsky Hamiltonian showing, specially, that Podolsky's generalised electrodynamics can be made stable by a proper choice of coupling constants.

Podolsky's theory has been developed by Bopp ${ }^{18}$ and independently by Podolsky and Schwed ${ }^{19}$ as a second-order gauge theory for the electromagnetic field in order to treat the $r^{-1}$ dependency in the electrostatic potential. Because of this behavior, the energy necessary to assemble a system of point charges has infinite contribution of the charge's self-energy. This problem is related to divergences in the infrared sector of the quantum electrodynamics (QED) as well to the problem of infinite vacuum polarisation current. The theory proposed is described by the Lagrangian density

$$
L=-\frac{1}{4} F_{\mu \nu} F^{\mu \nu}+\frac{1}{2} a^{2} \partial_{\lambda} F^{\mu \lambda} \partial^{\gamma} F_{\mu \gamma}
$$

with $F_{\mu \nu}=\partial_{\mu} A_{\nu}-\partial_{v} A_{\mu}$ as the components of the electromagnetic tensor field, and $a$ being a parameter with the dimension of the inverse of mass. The second-order derivative term in (1) results in a well defined electrostatic potential for $r=0$, so the self-energy contribution may be computed; it is just proportional to $q^{2} / a$ for each charge $q$. The theory may be interpreted as an effective theory for short distances, ${ }^{20}$ as a way to get rid of the problems related to the $r=0$ singularity in QED. In this case, the parameter $a$ becomes a cutoff distance for the theory. When $r \gg a$, the theory would become experimentally indistinguishable from Maxwell's theory. In this point of view, $a$ is linked to an effective radius for the electron.

If interpreted as a fundamental theory, the parameter $a$ is related to a sector of massive photons, which can be seen by the dispersion relation

$$
p^{2}\left(p^{2}-m_{\gamma}^{2}\right)=0, \quad p^{2} \equiv p_{\mu} p^{\mu}, \quad m_{\gamma}^{2} \equiv 1 / a^{2},
$$

taken from the generalised wave equation, result of the field equations of Lagrangian (1). This relation indicates two kinds of photons with modes $p^{2}=0$ and $p^{2}=m_{\gamma}^{2}$, respectively. The first mode corresponds to massless photons, and the second mode is linked to photons with mass parameter $m_{\gamma}$. Massive photons are not observed in nature, so it is not generally believed that Podolsky's theory is fundamental. Yet, experimental attempts to provide upper limits to $m_{\gamma}$ are taken from time to time. ${ }^{21}$ The best result, however, is given by Luo et al., ${ }^{22}$ which has $m_{f}<2.1 \times 10^{-51} \mathrm{~g}$.

In Sec. III, we discuss forms of relativistic dynamics, especially how to project generators of the Poincare group when a given form of dynamics is defined in Minkowski space-time. The first attempt of quantisation of Podolsky's field was made in instant-form, where time is defined by the $x^{0}$ axis as the evolution parameter. There are five different forms of such Hamiltonian dynamics, each one related to different decompositions of the Poincare group. ${ }^{23}$ The dynamics on the null-plane, also called the front-form dynamics, is the Hamiltonian dynamics of fields over a null-plane $x^{0}+x^{3}$ $=c t e$. The evolution parameter is chosen to be the coordinate $x^{+} \equiv \frac{1}{\sqrt{2}}\left(x^{0}+x^{3}\right)$, where the classical (quantum) evolution of the system is given by the definition of appropriate fundamental "equal-time" brackets (commutators), defined on a null-plane of constant $x^{+}$, plus a special set of initial-boundary data. 
There are some good reasons, both physical and mathematical, to analyse field theories on the null-plane. One of them is the fact that this kind of dynamics usually reduces the number of independent degrees of freedom necessary to describe a field. ${ }^{24,25}$ This is closely related to the fact that the stability group of the Poincare group in front-form, which is the sub-group of transformations that relates field configurations in a single surface $x^{+}=c t e$, has seven generators, one more than the six kinematic generators in instant-form. Besides, the algebra of these kinematic generators takes its simplest form in front-form dynamics. For some important systems, this feature is responsible for a complete separation of physical degrees of freedom, resulting in a clean and excitation-free quantum vacuum.

We leave the application of the null-plane HJ formalism for Sec. IV. The canonical structure of Podolsky's theory was already studied in Ref. 26, in instant-form, and in Ref. 27 on the null-plane, both using Dirac's method. However, these papers had the goal of analysing proper gauge conditions for the theory in order to clarify the physical degrees of freedom. Our main purpose here, using the HJ theory, is to obtain a complete set of involutive generators or Hamiltonians. We see that the presence of a non-involutive subset of constraints demands modification of the dynamics of the system, with the introduction of generalised brackets (GBs). With these brackets, we are able to find the complete set of generators, which close a Lie algebra with the GBs, assuring integrability as stated by Frobenius' theorem. On the other hand, any complete set of Hamiltonians generate canonical transformations, so we also present a way of defining these transformations and relate them to the gauge transformations of Podolsky's Lagrangian. This is done without any mention to Dirac's conjecture or even the necessity of ad hoc methods, such as the case of Castellani's procedure. ${ }^{28}$ In Sec. V, we present our final remarks.

\section{THE HJ THEORY FOR SINGULAR SYSTEMS AND HIGHER-ORDER ACTIONS}

\section{A. Higher-order theories}

Let us start with a system described by $n$ generalised coordinates $q^{i}$. In general, the coordinates should be at least of class $C^{2 k}$ in a time parameter $\tau$, but for now we may define the variables $q_{I}^{i}$ as the $I$ th derivative of $q^{i}$ with respect to $\tau$, where $I=\{0,1, \ldots, k\}$ for a given $k \in \mathbb{N}$. A high-order Lagrangian theory, in this case of order $k$, is a theory described by the action

$$
A[\gamma]=\int_{\gamma} d \tau L\left[\tau, q_{I}^{i}(\tau)\right]
$$

which is a functional of curve segments $\gamma: q^{i}=q^{i}(\tau)$ in the space of generalised coordinates.

Supposing $\delta \tau \equiv \bar{\tau}-\tau$ and $\delta q^{i} \equiv \bar{q}^{i}(\bar{\tau})-q^{i}(\tau)$ general first-order variations of the time and coordinates, we have the first-order variation

$$
\delta A=\int d \tau\left[\frac{\delta L}{\delta q^{i}}\left(\delta-\delta \tau \frac{d}{d \tau}\right) q^{i}+\frac{d}{d \tau}\left(\sum_{I=0}^{k-1} \frac{\delta L}{\delta q_{(I+1)}^{i}} \delta q_{I}^{i}-H \delta \tau\right)\right],
$$

where

$$
\frac{\delta L}{\delta q^{i}} \equiv \sum_{I=0}^{k}(-1)^{I} \frac{d^{I}}{d \tau^{I}} \frac{\partial L}{\partial q_{I}^{i}}
$$

are Lagrange derivatives, and

$$
H \equiv \sum_{I=0}^{k-1} \frac{\delta L}{\delta q_{(I+1)}^{i}} q_{(I+1)}^{i}-L
$$

is defined as the Hamiltonian function of the system. Unless stated otherwise, repeated lowercase latin indices carry implicit summation. Repeated uppercase latin indices, however, always carry explicit summation. 
Lagrange derivatives often appear to replace partial derivatives in Lagrangian systems with higher-order derivatives. For example, if the Lagrangian has the variables $\left(q^{i}, \dot{q}^{i}, \ddot{q}^{i}\right)$, Eq. (5) yields

$$
\frac{\delta L}{\delta q^{i}}=\frac{\partial L}{\partial q^{i}}-\frac{d}{d \tau}\left(\frac{\partial L}{\partial \dot{q}^{i}}\right)+\frac{d^{2}}{d \tau^{2}}\left(\frac{\partial L}{\partial \ddot{q}^{i}}\right)
$$

We can also derive a function with respect to any higher-order variable, which is the case of Lagrange derivatives inside the total time derivative in (4). For example, we have

$$
\frac{\delta L}{\delta q_{1}^{i}}=\frac{\partial L}{\partial q_{1}^{i}}-\frac{d}{d \tau}\left(\frac{\partial L}{\partial q_{2}^{i}}\right)+\frac{d^{2}}{d \tau^{2}}\left(\frac{\partial L}{\partial q_{3}^{i}}\right)+\cdots+(-1)^{k-1} \frac{d^{k-1}}{d \tau^{k-1}}\left(\frac{\partial L}{\partial q_{k}^{i}}\right),
$$

which leads to the general relation

$$
\frac{\delta L}{\delta q_{I}^{i}}=\sum_{J=I}^{k}(-1)^{J-I} \frac{d^{J-I}}{d \tau^{J-I}} \frac{\partial L}{\partial q_{J}^{i}},
$$

for a Lagrangian dependent on derivatives up to order $k$. Of course, the Lagrange derivative with respect to the highest order is equal to its partial derivative,

$$
\frac{\delta L}{\delta q_{k}^{i}}=\frac{\partial L}{\partial q_{k}^{i}}
$$

Back to the variational problem at hand, if the action $A[\gamma]$ has an extreme configuration $\gamma_{0}$, this configuration is a solution of the Euler-Lagrange (EL) equations

$$
\frac{\delta L}{\delta q^{i}}=0
$$

which are the equations of motion of action (3). The EL equations (8) are actually linear in the 2k-th derivative $q_{2 k}^{i}$, which is the reason for our first assumption that $q^{i}$ should be at least of class $C^{2 k}$.

\section{B. Equivalent Lagrangians}

The HJ formalism in Carathéodory's point of view ${ }^{1}$ requires a way of defining equivalent actions of functional (3). This is done by supposing a point-transformation $q^{i} \rightarrow q^{\prime i}$ with generating function $S\left(\tau, q_{I^{\prime}}^{i}\right)$. With this transformation, the new Lagrangian is related to the old one by $L^{\prime}=L-d S / d \tau$. Here we introduce another index $I^{\prime}=\{0,1, \ldots, k-1\}$ since it will become clear later that $S$ cannot depend on $q_{k}^{i}$. Adding a total derivative to the Lagrangian does not change the equations of motion, so if $\gamma_{0}$ is an extreme configuration of the action $\int L d \tau$, it is also an extreme configuration of the transformed action $\int L^{\prime} d \tau$. If this point-transformation leads to $L^{\prime}=0$ for $\gamma=\gamma_{0}$, and $L^{\prime}>0$ or $L^{\prime}<0$ for any $\gamma \neq \gamma_{0}$ in a close neighbourhood of $\gamma_{0}, A[\gamma]$ has, respectively, a local minimum $\left(L^{\prime}>0\right)$ or a local maximum $\left(L^{\prime}<0\right)$ in $\gamma_{0}$. Expanding $L^{\prime}$ in a Taylor series around $\gamma_{0}$, we see that the new Lagrangian is approximated by a lower-order term that is quadratic positive (or negative) definite in $\delta q_{I}^{i}$. The necessary condition for the extreme configuration become

$$
\left.\frac{\partial L^{\prime}}{\partial q_{I}^{i}}\right|_{\gamma=\gamma_{0}}=0
$$

which results in

$$
\frac{\partial S}{\partial q_{I^{\prime}}^{i}}=\frac{\partial L}{\partial q_{\left(I^{\prime}+1\right)}^{i}}-\frac{d}{d \tau}\left(\frac{\partial S}{\partial q_{\left(I^{\prime}+1\right)}^{i}}\right)
$$

at $\gamma=\gamma_{0}$.

If we take $I^{\prime}=k-1,(10)$ becomes

$$
\frac{\partial S}{\partial q_{(k-1)}^{i}}=\frac{\partial L}{\partial q_{k}^{i}}
$$


For $I^{\prime}=k-2$, we have

$$
\frac{\partial S}{\partial q_{(k-2)}^{i}}=\frac{\partial L}{\partial q_{(k-1)}^{i}}-\frac{d}{d \tau}\left(\frac{\partial S}{\partial q_{(k-1)}^{i}}\right)
$$

With (11), the result is

$$
\frac{\partial S}{\partial q_{(k-2)}^{i}}=\frac{\partial L}{\partial q_{(k-1)}^{i}}-\frac{d}{d \tau} \frac{\partial L}{\partial q_{k}^{i}}=\frac{\delta L}{\delta q_{(k-1)}^{i}},
$$

where we recognise the Lagrange derivative on the right. Following this iterative process, the general relations are found to be

$$
\frac{\partial S}{\partial q_{I^{\prime}}^{i}}=\frac{\delta L}{\delta q_{\left(I^{\prime}+1\right)}^{i}} .
$$

On the other hand, $L^{\prime}=L-d S / d \tau=0$ immediately leads to

$$
L-\frac{\partial S}{\partial \tau}-\sum_{I^{\prime}} q_{\left(I^{\prime}+1\right)}^{i} \frac{\partial S}{\partial q_{I^{\prime}}^{i}}=0 .
$$

Therefore, the existence of a function $S\left(\tau, q_{I^{\prime}}^{i}\right)$ that obeys conditions (12) and (13) is a necessary condition for the existence of an extreme configuration of action (3).

\section{The HJ equation}

The HJ formalism may be reached if we make Eq. (13) a PDE for the function $S$. In principle, we expect solving this problem with expressions of the type $q_{\left(I^{\prime}+1\right)}^{i}=\phi_{\left(I^{\prime}+1\right)}^{i}\left(\tau, q_{I^{\prime}}^{i}, \partial S / \partial q_{I^{\prime}}^{i}\right)$ that would be taken from (12). This, however, is not the case. Let us take again expression (12) for $I^{\prime}=k-1$,

$$
\frac{\partial S}{\partial q_{(k-1)}^{i}}=\frac{\delta L}{\delta q_{k}^{i}}=\frac{\partial L}{\partial q_{k}^{i}}=\psi_{i}\left(\tau, q_{I^{\prime}}^{i}, q_{k}^{i}\right)
$$

where $\psi$ is a function of $\tau$ and the variables $q_{I}^{i}$. This equation can indeed be inverted to produce the expression

if the Hessian condition

$$
q_{k}^{i}=\phi_{k}^{i}\left(\tau, q_{I^{\prime}}^{i}, \frac{\partial S}{\partial q_{(k-1)}^{i}}\right) \text {, }
$$

$$
\operatorname{det}\left(\frac{\partial \psi_{i}}{\partial q_{k}^{j}}\right)=\operatorname{det}\left(\frac{\partial^{2} L}{\partial q_{k}^{i} \partial q_{k}^{j}}\right) \neq 0
$$

is satisfied.

However, Eq. (12) for $I^{\prime}=k-2$ yields

$$
\frac{\partial S}{\partial q_{(k-2)}^{i}}=\frac{\partial L}{\partial q_{(k-1)}^{i}}-\frac{\partial^{2} L}{\partial \tau \partial q_{k}^{i}}-\sum_{I^{\prime}} q_{\left(I^{\prime}+1\right)}^{j} \frac{\partial^{2} L}{\partial q_{I^{\prime}}^{j} \partial q_{k}^{i}}-q_{(k+1)}^{j} \frac{\partial^{2} L}{\partial q_{k}^{j} \partial q_{k}^{i}},
$$

where a derivative superior to $k$ appears in the last term. This equation provides

$$
q_{(k+1)}^{j}=\phi_{(k+1)}^{j}\left(\tau, q_{I^{\prime}}^{i}, \frac{\partial S}{\partial q_{(k-1)}^{i}}, \frac{\partial S}{\partial q_{(k-2)}^{i}}\right),
$$

if, again, (15) is satisfied. All orders have the same behavior. Particularly, the lowest order $I^{\prime}=0$ results in

$$
q_{(2 k)}^{i}=\phi_{(2 k)}^{i}\left(\tau, q_{I^{\prime}}^{i}, \frac{\partial S}{\partial q_{I^{\prime}}^{i}}\right)
$$

Therefore, the best we can do is to invert (12) to obtain expressions for the variables of the theory from order $k$ to $2 k$. The condition for that is that the Hessian matrix

$$
W_{i j} \equiv \frac{\partial^{2} L}{\partial q_{k}^{i} \partial q_{k}^{j}}
$$


should be non-singular.

This is not a problem if we consider the variables $q_{I^{\prime}}^{i}$ independent coordinates of a configuration space $\mathbb{Q}$. This interpretation is also suggested by the end-point term in (4), which defines the structure of the canonically conjugated variables of the theory: that sum is a linear combination of independent variations $\delta q_{I^{\prime}}^{i}$ only up to order $I=k-1$ since $L$ depends on the coordinates up to order $k$. In this case, (13) is just written by

$$
\frac{\partial S}{\partial \tau}+\sum_{I^{\prime}} \frac{\partial S}{\partial q_{\left(I^{\prime}-1\right)}^{i}} q_{I^{\prime}}^{i}+\frac{\partial S}{\partial q_{(k-1)}^{i}} \phi_{k}^{i}-L\left[\tau, q_{I^{\prime}}^{i}, \phi_{k}^{i}\right]=0,
$$

and no other equation is needed.

Now let us observe the Hamiltonian function defined in (6), which can be written using (12) by

$$
H=\sum_{I^{\prime}} \frac{\delta L}{\delta q_{I^{\prime}}^{i}} q_{I^{\prime}}^{i}+\frac{\delta L}{\delta q_{k}^{i}} \phi_{k}^{i}-L=\sum_{I^{\prime}} \frac{\partial S}{\partial q_{\left(I^{\prime}-1\right)}^{i}} q_{I^{\prime}}^{i}+\frac{\partial S}{\partial q_{(k-1)}^{i}} \phi_{k}^{i}-L
$$

for $I^{\prime}>0$. Substituting (14), we may write (19) as

$$
\frac{\partial S}{\partial \tau}+H\left(\tau, q_{I^{\prime}}^{i}, \frac{\partial S}{\partial q_{I^{\prime}}^{i}}\right)=0 .
$$

Equation (21) is the expected Hamilton-Jacobi equation.

The introduction of canonical variables is straightforward. In the $\mathrm{HJ}$ formalism, the momenta conjugated to the variables $q_{I^{\prime}}^{i}$ are defined as the components of the gradient of the function $S$,

$$
p_{i}^{I^{\prime}} \equiv \frac{\partial S}{\partial q_{I^{\prime}}^{i}}=\frac{\delta L}{\delta q_{\left(I^{\prime}+1\right)}^{i}} .
$$

This definition results in the canonical Hamiltonian

$$
H_{0}\left(\tau, q_{I^{\prime}}^{i}, p_{i}^{I^{\prime}}\right) \equiv \sum_{I^{\prime}} p_{i}^{\left(I^{\prime}-1\right)} q_{I^{\prime}}^{i}+p_{i}^{(k-1)} \phi_{k}^{i}-L,
$$

with all $q_{k}^{i}$ substituted by $\phi_{k}^{i}$ as functions of the momenta. It also results in the canonical HJ equation

$$
\Phi_{0}\left(\tau, p_{\tau}, q_{I^{\prime}}^{i}, p_{i}^{I^{\prime}}\right) \equiv \pi_{0}+H_{0}\left(\tau, q_{I^{\prime}}^{i}, p_{i}^{I^{\prime}}\right)=0
$$

where we introduce the notation $\pi_{0} \equiv \partial S / \partial \tau$.

\section{The HJ equations for singular systems}

The Hessian condition appears in the HJ formalism as a condition for the existence of PDE (21). But in the case of violation of the Hessian condition (15), which implies the existence of constraints, it is still straightforward to define a HJ approach. Let us suppose the $n \times n$ Hessian matrix $W_{i j}$ to be singular of rank $m<n$. Then, there is a regular sub-matrix $m \times m$ and a null-space of dimension $r=n-m$, splitting the configuration space in two subspaces: the space of the variables $q_{I^{\prime}}^{a}$, for $a=\{1, \ldots, m\}$, which will be called $\mathbb{Q}_{m}$, and the space of the variables $t_{I^{\prime}}^{z} \equiv q_{I^{\prime}}^{z}$ with $z=\{1, \ldots, r\}$, which will be called $\Gamma_{r}$. The former are variables belonging to the regular space of the Hessian, related to the matrix

$$
W_{a b} \equiv \frac{\partial^{2} L}{\partial q_{k}^{a} \partial q_{k}^{b}},
$$

which obeys det $W_{a b} \neq 0$. The latter are variables belonging to the null-space of the same Hessian matrix.

In the singular case, Eq. (12) produces the $m \cdot k$ relations

$$
q_{\left(k+I^{\prime}\right)}^{a}=\phi_{\left(k+I^{\prime}\right)}^{a}\left(\tau, t_{I^{\prime}}^{z}, q_{I^{\prime}}^{a}, p_{a}^{I^{\prime}}\right), \quad p_{a}^{I^{\prime}} \equiv \frac{\partial S}{\partial q_{I^{\prime}}^{a}}
$$

and also the $r \cdot k$ identities

$$
\frac{\partial S}{\partial t_{I^{\prime}}^{z}}=\frac{\delta L}{\delta q_{\left(I^{\prime}+1\right)}^{z}} \equiv-H_{z}^{I^{\prime}}\left(\tau, t_{I^{\prime}}^{z}, q_{I^{\prime}}^{a}, p_{a}^{I^{\prime}}\right)
$$


In canonical form, (27) becomes

$$
\Phi_{z}^{I^{\prime}}\left(\tau, t_{I^{\prime}}^{y}, q_{I^{\prime}}^{a}, \pi_{y}^{I^{\prime}}, p_{a}^{I^{\prime}}\right) \equiv \pi_{z}^{I^{\prime}}+H_{z}^{I^{\prime}}=0, \quad \pi_{z}^{I^{\prime}} \equiv \frac{\partial S}{\partial t_{I^{\prime}}^{z}} .
$$

Equations (28) form a set of $r \cdot k$ canonical constraints and also a set of $r \cdot k$ first-order PDEs.

We notice that the canonical Hamiltonian function (20) does not depend on the variables $t_{k}^{z}$, so the definition of this function is not dependent on the Hessian condition, and the HJ equation (24) is still valid. Let us introduce the notation $t_{I^{\prime}}^{\alpha}=\left(\tau, t_{I^{\prime}}^{z}\right)$, then $\alpha=\{0,1, \ldots, r\}$. Since the momentum conjugated to $\tau$ is named $\pi_{0}$, we may also include these as new variables in the theory as $\pi_{\alpha}^{I^{\prime}}=\left(\pi_{0}, \pi_{z}^{I^{\prime}}\right)$. Then, Eqs. (24) and (28) may be written in a unified way,

$$
\Phi_{\alpha}^{I^{\prime}}\left(t_{I^{\prime}}^{\beta}, q_{I^{\prime}}^{a}, \pi_{\beta}^{I^{\prime}}, p_{a}^{I^{\prime}}\right) \equiv \pi_{\alpha}^{I^{\prime}}+H_{\alpha}^{I^{\prime}}\left(t_{I^{\prime}}^{\beta}, q_{I^{\prime}}^{a}, p_{a}^{I^{\prime}}\right)=0,
$$

where we also use $H_{\alpha}^{I^{\prime}}=\left(H_{0}, H_{z}^{I^{\prime}}\right)$. Equation (29) forms a set of Hamilton-Jacobi first-order partial differential equations. Summation is also implicit whenever a greek index appears double.

\section{E. Integrability and characteristic equations}

The HJ equation (29) is necessary but still not sufficient for the existence of extreme configurations of the action $A[\gamma]$. It is still necessary that (29) provides at least one complete solution for the $S$ function. These equations are generally a set of $(r+1) \cdot k$ non-linear coupled PDEs of the first-order, so we expect that a complete solution contains $(r+1) \cdot k$ constants of integration related to $(r+1) \cdot k$ linearly independent parameters. In other words, we expect that a complete solution has the form $S=S\left[t_{I^{\prime}}^{\alpha}, q_{I^{\prime}}^{a}\left(t_{I^{\prime}}^{\alpha}\right)\right]$ for a set of $(r+1) \cdot k$ parameters $t_{I^{\prime}}^{\alpha}$ and a set of $m \cdot k$ variables $q_{I^{\prime}}^{a}\left(t_{I^{\prime}}^{\alpha}\right)$. Of course, this is possible only if $\Phi_{\alpha}^{I^{\prime}}$ form a set of $(r+1) \cdot k$ linearly independent equations. For the following, there is no need to carry the $I^{\prime}$ index. We just write the generators $\Phi_{\alpha} \equiv \Phi_{\alpha}^{I^{\prime}}$, in which now $\alpha=0, \ldots,(r+1) \cdot k$, and use the compact notations $q^{i} \equiv\left(t_{I^{\prime}}^{\alpha}, q_{I^{\prime}}^{a}\right)=\left(t^{\alpha}, q^{a}\right)$ and $p_{i} \equiv\left(\pi_{\alpha}^{I^{\prime}}, p_{a}^{I^{\prime}}\right)=\left(\pi_{\alpha}, p_{a}\right)$.

The necessary and sufficient condition for complete integrability is Frobenius' integrability condition ${ }^{7}$

$$
\left\{\Phi_{\alpha}, \Phi_{\beta}\right\}=C_{\alpha \beta}^{\gamma} \Phi_{\gamma}
$$

in which $C_{\alpha \beta}^{\gamma}$ is a set of structure coefficients. The brackets are the complete Poisson brackets (PB),

$$
\{A, B\} \equiv \frac{\partial A}{\partial q^{i}} \frac{\partial B}{\partial p_{i}}-\frac{\partial B}{\partial q^{i}} \frac{\partial A}{\partial p_{i}} .
$$

If (30) holds, $\Phi_{\alpha}$ form a complete set of constraints in involution with PB operation. We name these set involutive constraints. Therefore, Frobenius' conditions are resumed in the fact that $\Phi_{\alpha}$ are generators of a Lie algebra with the PB.

If $F\left(q^{i}, p_{i}\right)$ is an observable of the complete phase-space, its dynamics is given by the fundamental differential

$$
d F=\left\{F, \Phi_{\alpha}\right\} d t^{\alpha},
$$

so the dynamics takes place in the complete phase-space of the variables $\xi^{i} \equiv\left(t_{I^{\prime}}^{\alpha}, q_{I^{\prime}}^{a}, \pi_{\alpha}^{I^{\prime}}, p_{a}^{I^{\prime}}\right)$, where $\Phi_{\alpha}^{I^{\prime}}(\xi)$ are the generators and $t_{I^{\prime}}^{\alpha}$ are the evolution parameters. This phase-space is actually degenerate. Let us calculate (now with explicit indices $I^{\prime}$ )

$$
d q_{I^{\prime}}^{a}=\sum_{J^{\prime}}\left\{q_{I^{\prime}}^{a}, \Phi_{\alpha}^{J^{\prime}}\right\} d t_{J^{\prime}}^{\alpha}=\sum_{J^{\prime} K^{\prime}}\left\{q_{I^{\prime}}^{a}, p_{b}^{K^{\prime}}\right\} \frac{\partial \Phi_{\alpha}^{J^{\prime}}}{\partial p_{b}^{K^{\prime}}} d t_{J^{\prime}}^{\alpha} .
$$

Using definition (31), we see that $\left\{q_{I^{\prime}}^{a}, p_{b}^{K^{\prime}}\right\}=\delta_{b}^{a} \delta_{I^{\prime}}^{K^{\prime}}$, so

$$
d q_{I^{\prime}}^{a}=\sum_{J^{\prime}} \frac{\partial \Phi_{\alpha}^{J^{\prime}}}{\partial p_{a}^{I^{\prime}}} d t_{J^{\prime}}^{\alpha}
$$


On the other hand,

$$
d p_{a}^{I^{\prime}}=\sum_{J^{\prime}}\left\{p_{a}^{I^{\prime}}, \Phi_{\alpha}^{J^{\prime}}\right\} d t_{J^{\prime}}^{\alpha}=\sum_{J^{\prime} K^{\prime}}\left\{p_{a}^{I^{\prime}}, q_{K^{\prime}}^{b}\right\} \frac{\partial \Phi_{\alpha}^{J^{\prime}}}{\partial q_{K^{\prime}}^{b}} d t_{J^{\prime}}^{\alpha}=-\sum_{J^{\prime}} \frac{\partial \Phi_{\alpha}^{J^{\prime}}}{\partial q_{I^{\prime}}^{a}} d t_{J^{\prime}}^{\alpha} .
$$

Equations (34) and (35) are the generalisations of Hamilton's equations for singular systems. They are generally called the characteristic equations (CEs) of system (29). The third fundamental characteristic equation is given by

$$
d S=\sum_{I^{\prime}}\left\{S, \Phi_{\alpha}^{I^{\prime}}\right\} d t_{I^{\prime}}^{\alpha}=\sum_{I^{\prime}}\left(p_{a}^{I^{\prime}} d q_{I^{\prime}}^{a}+\pi_{\alpha}^{I^{\prime}} d t_{I^{\prime}}^{\alpha}-\Phi_{\alpha}^{I^{\prime}} d t_{I^{\prime}}^{\alpha}\right)
$$

and results in integral equations for $S$ if (34) and (35) provide solutions for $p_{a}^{I^{\prime}}\left(t_{I^{\prime}}^{\alpha}\right)$ and $q_{I^{\prime}}^{a}\left(t_{I^{\prime}}^{\alpha}\right)$. The CEs for $t_{I^{\prime}}^{\alpha}$ and $\pi_{\alpha}^{I^{\prime}}$ are just identities, which reveals the degenerate character of the complete phase space.

There is, however, a reduced phase-space that is not degenerate. It is the space of the variables $\xi^{A} \equiv\left(q_{I^{\prime}}^{a}, p_{a}^{I^{\prime}}\right)$. In fact, it is shown in Ref. 7 that the sector of the variables $\left(t_{I^{\prime}}^{\alpha}, \pi_{\alpha}^{I^{\prime}}\right)$ in the complete phase space has a zero volume element. The reason for that is the fact that $t_{I^{\prime}}^{\alpha}$ form a parameter space that is isomorphic to a complete affine vector space. In other words, the solutions of the characteristic equations are trajectories in the reduced phase-space, of the type $\xi^{A}=\xi^{A}\left(t_{I^{\prime}}^{\alpha}\right)$, parametrised by the variables $t_{I^{\prime}}^{\alpha}$. For this reason, we call $\xi^{A}$ the dependent variables and $t_{I^{\prime}}^{\alpha}$ the independent variables of the theory. Usually, the dependent variables are related to the "true" degrees of freedom of a physical system.

\section{F. The generalised brackets}

The Frobenius theorem implies that the HJ equations $\Phi_{\alpha}=0$ must be complete and linearly independent. However, in physical examples, this condition is not usually satisfied. If the set $\Phi=\left\{\Phi_{\alpha}\right\}$ is not integrable, two possible reasons are the following: (1) there may be other HJ equations not included in this set, a case in which the equations are not complete and (2) a subset of $\Phi$ is not linearly independent. In this case, since a physical system must be integrable, the aim is to discover a complete set of involutive constraints. For that, we use the procedure outlined in Ref. 6.

If the HJ equations $\Phi_{\alpha}=0$ are valid, Frobenius' condition $\{\Phi, \Phi\} \subset \Phi$ implies that the generators are dynamical invariants, in other words,

$$
d \Phi_{\alpha}=\left\{\Phi_{\alpha}, \Phi_{\beta}\right\} d t^{\beta}=0 .
$$

Equation (37) is a set of linear equations for the differential of the independent variables,

$$
M_{\alpha \beta} d t^{\beta}=0,
$$

where we introduced the matrix $M_{\alpha \beta} \equiv\left\{\Phi_{\alpha}, \Phi_{\beta}\right\}$. Remember that, in our case, this matrix has dimension $(r+1) \cdot k$.

We suppose the general case in which $M_{\alpha \beta}$ has rank $p \leq(r+1) \cdot k$. Then, there is an invertible sub-matrix $M_{x y}$ whose entries are the PB between a subset $\left\{\Phi_{x}\right\} \subset \Phi$, with $x=1, \ldots, p$. In this case, for $\alpha=x$ (38) becomes

$$
M_{x \beta} d t^{\beta}=0 \Longrightarrow M_{x y} d t^{y}=-M_{x \alpha^{\prime}} d t^{\alpha^{\prime}},
$$

in which $\alpha^{\prime}=1, \ldots, q$ is the index of the null-space of $M_{\alpha \beta}$. Then, (38) can be written as

$$
d t^{x}=-\left(M^{-1}\right)^{x y}\left\{\Phi_{y}, \Phi_{\alpha^{\prime}}\right\} d t^{\alpha^{\prime}},
$$

where $\left(M^{-1}\right)^{x y}$ is the inverse matrix related to $M_{x y}$. Therefore, if the set $\Phi$ is not integrable, the former independent variables $t^{\alpha} \equiv\left(t^{x}, t^{\alpha^{\prime}}\right)$ are not mutually independent due to the fact that the matrix $M_{\alpha \beta}$ has non-zero rank. In fact, (40) can be written for any set of non-involutive constraints, not only in the case of maximal rank.

Let us write (38) for $\alpha=\alpha^{\prime}$,

$$
M_{\alpha^{\prime} \beta} d t^{\beta}=0 \Longrightarrow M_{\alpha^{\prime} x} d t^{x}+M_{\alpha^{\prime} \beta^{\prime}} d t^{\beta^{\prime}}=0
$$


Using (40), we have

$$
\left[\left\{\Phi_{\alpha^{\prime}}, \Phi_{\beta^{\prime}}\right\}-\left\{\Phi_{\alpha^{\prime}}, \Phi_{x}\right\}\left(M^{-1}\right)^{x y}\left\{\Phi_{y}, \Phi_{\beta^{\prime}}\right\}\right] d t^{\beta^{\prime}}=0 .
$$

Now we introduce the generalised brackets (GBs) between two observables $A$ and $B$,

$$
\{A, B\}^{*} \equiv\{A, B\}-\left\{A, \Phi_{x}\right\}\left(M^{-1}\right)^{x y}\left\{\Phi_{y}, B\right\} .
$$

Then we may write (41) as

$$
\left\{\Phi_{\alpha^{\prime}}, \Phi_{\beta^{\prime}}\right\}^{*} d t^{\beta^{\prime}}=0
$$

It is straightforward to show that the GBs are good brackets. They are anti-symmetric bilinear differential operators that obey the Jacobi identity.

Suppose we have chosen a subset $\left\{\Phi_{x}\right\} \subset \Phi$ that is not of maximal rank. In this case, (44) again yields two sets of equations, one of them will give rise to another generalised bracket since not all linear combinations of the independent variables were discovered. The other set is just (44) again. When all linear combinations of the parameters are eliminated by a final GB, the differentials $d t^{\alpha^{\prime}}$ in (44) are truly linearly independent. Then, the integrability conditions are just given by

$$
\left\{\Phi_{\alpha^{\prime}}, \Phi_{\beta^{\prime}}\right\}^{*}=0 .
$$

Equation (45) may give two results. It may be identically satisfied for some generators, but it may result in relations between the phase-space variables, which should be taken as new constraints. If found, these new HJ equations should be added to the set $\Phi^{\prime} \equiv\left\{\Phi_{\alpha^{\prime}}\right\}$. Equation (44) should be used again since it is possible that the new set $\Phi^{\prime}$ is non-involutive, and a new GB may be defined. This process must be repeated until (45) gives no more constraints, completing the system of generators, which will happen if the system is really integrable. In this case, the remaining set of constraints obeys the condition

$$
\left\{\Phi_{\alpha^{\prime}}, \Phi_{\beta^{\prime}}\right\}^{*}=C_{\alpha^{\prime} \beta^{\prime}}{ }^{\gamma^{\prime}} \Phi_{\gamma^{\prime}},
$$

this time with the GB. Therefore, if the system is integrable, we must be able to reduce it to a set of complete involutive constraints with a GB.

With a complete involutive set, the fundamental differential of an observable $F$ is given by

$$
d F=\left\{F, \Phi_{\alpha}\right\} d t^{\alpha}=\left\{F, \Phi_{\alpha^{\prime}}\right\}^{*} d t^{\alpha^{\prime}},
$$

with the use of (40). Note that the generators belonging to the invertible part of the matrix $M_{\alpha \beta}$, the ones that are not involutive, are now completely eliminated since the GB of a non-involutive constraint with any observable of the phase-space is identically zero. If a system has non-involutive constraints, the characteristic equations must be calculated from (47). Therefore, a subset of non-involutive constraints is responsible for modifying the dynamics of the system.

\section{G. Canonical and Lagrangian symmetries}

If $\Phi$ is a complete set of involutive constraints, it is shown in Ref. 7 that each member of the set is a generator of an active canonical transformation on the reduced phase-space, called a characteristic flow $(\mathrm{CF})$. This is better seen in a geometrical framework: the symplectic structure of the complete phase-space is a degenerate 2 -form $\omega=-d \theta$, where $\theta=d S$ is the fundamental lepagian 1 -form defined by CE (36). The symplectic form is degenerate since it can be written as the sum of a regular 2-form $\omega_{P}=d q^{a} \wedge d p_{a}$, in canonical coordinates, and a singular 2-form $a=d t^{\alpha} \wedge d \pi_{\alpha}$ when the HJ equations $\Phi_{\alpha}=0$ are satisfied. In fact, the 2-form $a$ is identically a zero form when Frobenius' condition (46) is satisfied. The 2-form $\omega_{P}$ defines the symplectic structure of the reduced phase-space.

A canonical transformation is any change in the phase-space that preserves its symplectic structure. To relate each $\Phi_{\alpha}$ to a canonical transformation, we define a vector field $X_{\alpha} \equiv\left\{\bullet, \Phi_{\alpha}\right\}^{*}$ to each $\Phi_{\alpha}$ (the $\bullet$ symbol takes the place of a phase-space observable). If the set $\Phi$ is completely integrable, the Lie algebra (46) of the generators implies a Lie algebra of the vector fields $X_{\alpha}$,

$$
\left[X_{\alpha}, X_{\beta}\right]=X_{\alpha} X_{\beta}-X_{\beta} X_{\alpha}=f_{\alpha \beta}^{\gamma} X_{\gamma},
$$

if the structure coefficients $f_{\alpha \beta}^{\gamma}=-C_{\alpha \beta}{ }^{\gamma}$ are independent of the complete phase-space variables. Supposing (48) holds, any operator with the form $T \equiv \exp \left(\Delta t^{\alpha} X_{\alpha}\right)$ is a member of the group of 
canonical transformations if, and only if, $[\omega, T]=0$, which actually is the case. Even if (48) is not obeyed, it is shown that $\left[X_{\alpha}, \omega\right]=0$, which implies that the symplectic structure is preserved by the infinitesimal first-order transformation

$$
\delta \xi=\delta t^{\alpha} X_{\alpha} \xi .
$$

We call (49) a characteristic flow generated by the vector fields $X_{\alpha}$. Note that CEs (34) and (35) themselves have the form of characteristic flows $d \xi=d t^{\alpha} X_{\alpha} \xi$. Therefore, the Frobenius theorem implies that each $\Phi_{\alpha}$ generates its own CF, related to an independent variable $t^{\alpha}$, and each of these flows is independent of the others. Moreover, a general CF is a linear combination of the flows generated by each $X_{\alpha}$, with form (49).

A special CF is defined as follows. Suppose $\delta t^{0}=\delta \tau=0$ and $\delta t^{z}=\epsilon^{z}$, where $\epsilon^{z}$ form a set of LI constant parameters. In this case,

$$
\delta \xi=\epsilon^{z} X_{z} \xi=\left\{\xi, \Phi_{z}\right\}^{*} \epsilon^{z} .
$$

Transformation (50) is a canonical flow if the generators $\Phi_{z}$ close themselves a Lie algebra $\left\{\Phi_{x}, \Phi_{y}\right\}^{*}$ $=C_{x y}{ }^{2} \Phi_{z}$. On the other hand, the only way to assure this algebra is if it is restricted to the reduced phase-space, where $\Phi_{\alpha}=0$ holds. In this case, all algebrae become abelian. Transformation (50) is restricted to the reduced phase-space where $\left\{\Phi_{x}, \Phi_{y}\right\}^{*}=0$ is a characteristic flow, which was called "point transformations" by Dirac in his Hamiltonian picture. ${ }^{3}$ The generator of the point transformation (50) is given by $G=\Phi_{z} \epsilon^{z}$ since $\delta \xi=\{\xi, G\}$ reproduces (50) when $\Phi_{z}=0$.

If the original system has Lagrangian symmetries, e.g., local gauge symmetries in field theories, we expect these symmetries manifest themselves as canonical symmetries, in this case, characteristic flows in the HJ picture. In this case, Eq. (4) for $\delta \tau=0$ yields the Lie equation

$$
\delta L=\frac{\delta L}{\delta q^{i}} \delta q^{i}+\frac{d}{d \tau}\left(\sum_{I^{\prime}} \frac{\delta L}{\delta q_{\left(I^{\prime}+1\right)}^{i}} \delta q_{I^{\prime}}^{i}\right)=0,
$$

and it has to be satisfied by the point transformation (50) for $\delta q^{i}=\epsilon^{z} X_{z} q^{i}=\left\{q^{i}, G\right\}^{*}$.

\section{FORMS OF RELATIVISTIC DYNAMICS}

\section{A. Poincaré generators in field theories}

In Sec. II, we revised the HJ theory for singular systems in a classical mechanical background. But since we propose the study of Podolsky's electrodynamics, our particular interest rests in second-order relativistic field theories, described by the functional

$$
A[\phi] \equiv \int_{\Omega} L\left[x^{\mu}, \phi^{a}(x), \phi_{\mu}^{a}(x), \phi_{\mu \nu}^{a}(x)\right] d \omega .
$$

In (52), $\Omega$ is the volume of an $(d+1)$-dimensional Minkowski space-time $\mathcal{M}$ spanned by a rectangular coordinate system $x^{\mu}=\left(x^{0}, x^{1}, x^{2}, \ldots, x^{d}\right)$ with volume element $d \omega \equiv d x^{0} d x^{1} \cdots d x^{d}$. The Lagrangian density $L$ depends generally on the space-time coordinates $x^{\mu}$, the fields $\phi^{a}(x)$ at each point $x \in \Omega$, and its first $\left(\phi_{\mu}^{a} \equiv \partial_{\mu} \phi^{a}\right)$ and second $\left(\phi_{\mu \nu}^{a} \equiv \partial_{\mu} \partial_{\nu} \phi^{a}\right)$ derivatives. If we are dealing with a relativistic field, $L$ must be Lorentz invariant. Action (52) is distinct from (3) because the domain is a set of fields of several variables, and the integral itself is a multiple integral. For this paper, the metric has a signature $(+---)$.

With (52), the natural path for a HJ formalism in Carathéodory's point of view would be to define equivalent actions. But unlike the case of classical mechanics, there is not a unique way of implementing Carathéodory's programme in a covariant way. The simplest method, introduced by Weyl,${ }^{29}$ is adding a simple boundary term to $A$. A second way, developed by Carathéodory himself, ${ }^{2}$ would be adding a determinant to the Lagrangian density. Each method leads to distinct definitions of geodesic fields, resulting in distinct HJ theories. Carathéodory's choice leads to a Hamiltonian function defined by the determinant of the energy-momentum tensor. Weyl's choice is the usual one because it is an analog to the classical mechanical method. Moreover, it does not suffer from important limitations of the alternative approach, e.g., the requirement of positivity of the Lagrangian 
density. But even if we choose Weyl's definition of geodesic fields, an important characteristic of relativistic theories makes impracticable the construction of a covariant $\mathrm{HJ}$ theory with constraints. These theories are invariant under any particular choice of the global evolution parameter or time. In this case, the HJ equation becomes an identity, not a PDE. An attempt to solve this problem is also done in Ref. 2, but it relies on unphysical assumptions if applied to fundamental fields. Until now, no satisfactory way of defining a covariant HJ formalism for constrained systems is at hand.

This problem is closely related to the problem of construction of covariant Hamiltonian formalisms with constraints. Dirac was the first to conclude that a consistent Hamiltonian approach for field theories requires a choice of relativistic dynamics. ${ }^{23}$ Such a choice is essentially a choice of "time" parameter $\tau$ as a linear combination of the space-time axes, defined in the direction of a vector field $u(x)$, defined at each space-time event $x$. This vector field may be related to the world line of a physical observer in a referential frame, but this is not necessarily the case: a given frame of reference can use different forms of dynamics to describe a physical system. Along with this vector field, one chooses a family $\Sigma$ of $d$-dimensional surfaces orthogonal to $u(x)$ at each point $x \in \Omega$. Each member $\Sigma_{\tau} \in \Sigma$ must be labeled uniquely by a value of $\tau$, then we must choose the family so that the world line of a given observer intersects each family member once.

When the vector field $u(x)$ and the family $\Sigma$ are chosen, the symmetry group of the spacetime may be decomposed. If $T_{\mu \nu}$ are the components of the energy-momentum tensor and the time evolution generator, the Hamiltonian function is defined by the double projection

$$
H \equiv \int_{\Sigma_{\tau}} d \sigma u_{\mu} T_{\nu}^{\mu} u^{\nu}
$$

where $d \sigma_{x}$ is the d-volume element of $\Sigma_{\tau}$. If $w$ is a unit vector orthogonal to $u$,

$$
P_{w} \equiv \int_{\Sigma_{\tau}} d \sigma u_{\mu} T_{\nu}^{\mu} w^{v}
$$

defines the linear momentum in the direction of $w$, which generates translations in $\Sigma_{\tau}$ in the $w$ direction.

Let $\left\{v_{i}(x)\right\}$ be a base of vector fields defined in $\Sigma_{\tau}$ for a given point $x$, where $i, j=1, \ldots, d$. In this case,

$$
L_{i j} \equiv \int_{\Sigma_{\tau}} d \sigma u_{\mu}\left(T_{\alpha}^{\mu} x_{\beta}-T_{\beta}^{\mu} x_{\alpha}\right) v_{i}^{\alpha} v_{j}^{\beta}
$$

is the orbital angular momentum matrix, and

$$
S_{i j} \equiv-\int_{\Sigma_{\tau}} d \sigma u_{\mu}\left[\frac{\delta L}{\delta \phi_{\mu}^{a}} \phi_{\nu}^{a}\left(J_{\alpha \beta}\right)_{\gamma}^{v} x^{\gamma}\right] v_{i}^{\alpha} v_{j}^{\beta}
$$

is the spin matrix of the fields, where $\left(J_{\alpha \beta}\right)_{\gamma}^{v}$ are the generators of the Lorentz group. The matrix $M_{i j}=L_{i j}+S_{i j}$ generates rotations in $\Sigma_{\tau}$. We also have boosts, generated by

$$
B_{j} \equiv \int_{\Sigma_{\tau}} d \sigma u_{\mu}\left[T_{\alpha}^{\mu} x_{\beta}-T_{\beta}^{\mu} x_{\alpha}-\frac{\delta L}{\delta \phi_{\mu}^{a}} \phi_{\nu}^{a}\left(J_{\alpha \beta}\right)_{\gamma}^{v} x^{\gamma}\right] u^{\alpha} v_{j}^{\beta}
$$

which are pseudo-rotations in the plane defined by $v_{j}$ and $u$.

\section{B. Null-plane dynamics}

Because of the causal structure of Minkowski's metric, there is not a unique way of defining a Hamiltonian dynamics. The most simple and used one was called by Dirac ${ }^{23}$ the instant-form dynamics. It is the dynamics of fields in 3-dimensional Euclidian spaces orthogonal to the time axis $t=x^{0} / c$, the time measured by a clock at rest with respect to the laboratory. In this case, $u$ is given by the components $(1,0,0,0)$, while the base vectors $v_{i}$ are the usual $\left\{x^{1}, x^{2}, x^{3}\right\}$ axes. The Hamiltonian function is given by $H=\int_{\Sigma_{\tau}} d \sigma T_{00}$, and the linear momenta are just $P_{i}=\int_{\Sigma_{\tau}} d \sigma T_{0 i}$. Generators of rotations and boosts are also immediately calculated from (55)-(57). 
The null-plane dynamics, on the other hand, is the form of dynamics where $u$ lies in the light-cone. We define time as the parameter

$$
x^{+}=\frac{1}{\sqrt{2}}\left(x^{0}+x^{3}\right)
$$

so $u$ takes the form of the axis

$$
u^{\mu}=\frac{1}{\sqrt{2}}(1,0,0,1) .
$$

The equation $x^{+}=$const. defines a characteristic hyper-surface $\Sigma_{x^{+}}$orthogonal to the $u$ axis. This surface is called the null-plane, and it represents an electromagnetic wave front in vacuum.

With this choice of the evolution parameter and characteristic surfaces, it is convenient to choose an appropriate coordinate system. Let $x^{\mu}$ be the rectangular coordinates $\left(x^{0}, x^{1}, x^{2}, x^{3}\right)$, and let us consider the following transformation:

$$
y^{\mu}=\Gamma_{\nu}^{\mu} x^{\nu},
$$

with

$$
\Gamma=\frac{1}{\sqrt{2}}\left(\begin{array}{cccc}
1 & 0 & 0 & 1 \\
1 & 0 & 0 & -1 \\
0 & \sqrt{2} & 0 & 0 \\
0 & 0 & \sqrt{2} & 0
\end{array}\right),
$$

in which $\mathbf{I}$ is the $2 \times 2$ identity matrix, and $y^{\mu}$ is defined by the set $\left(x^{+}, x^{-}, x^{1}, x^{2}\right)$ in this order. We see that $x^{ \pm}=1 / \sqrt{2}\left(x^{0} \pm x^{3}\right)$, while the remaining coordinates are unchanged. The coordinates $y^{\mu}$ are called null-plane coordinates.

The null-plane dynamics was mistaken, for some time, with a limiting process known as the infinite momentum frame. ${ }^{25}$ But transformation (60) is not a reference choice. It is actually a parameter choice with a combining coordinate system. It is not even a Lorentz transformation since the metric in $y$ is given by

$$
\eta=\left(\begin{array}{ccc}
0 & 1 & 0 \\
1 & 0 & 0 \\
0 & 0 & -\mathbf{I}_{2 \times 2}
\end{array}\right) .
$$

With this metric, the norm of a Lorentz vector is not of a quadratic form in the temporal (+) and longitudinal (-) components, but remains quadratic in the transverse components 1 and 2,

$$
A^{2}=\eta_{\mu \nu} A^{\mu} A^{v}=2 A^{+} A^{-}-\left(A^{i}\right)^{2}, \quad i=1,2 .
$$

We stress that metric (62) is the metric used to raise and lower indices in the null-plane coordinates.

For example, the d'Alambertian operator assumes the form

$$
\square \equiv \partial_{\mu} \partial^{\mu}=\partial_{+} \partial^{+}+\partial_{-} \partial^{-}-\nabla^{2}=2 \partial_{+} \partial_{-}-\nabla^{2},
$$

where $\nabla \equiv \partial_{i} \partial_{i}$. In this case, the null-plane dynamics is distinct from the usual instant-form. The Klein-Gordon equation, for instance,

$$
\left(\square+m^{2}\right) \phi=0,
$$

has the null-plane form

$$
\partial_{+} \partial_{-} \phi=\frac{1}{2}\left(\nabla^{2}-m^{2}\right) \phi
$$

This is a first-order equation in the temporal coordinate $x^{+}$; therefore, the initial value problem is not a Cauchy problem but a characteristic value problem. To fix a unique solution, it is necessary to provide a field configuration in a plane $x^{+}=$const. and a second configuration in some $x^{-}=$const . plane, for example.

In the null-plane, we may write expressions for the Hamiltonian function and the linear momenta based on Eqs. (53) and (54). The $x^{+}$evolution generator is given by

$$
H=\int_{\Sigma} d \sigma T_{+}^{+}=\int_{\Sigma} d \sigma T_{-+}, \quad d \sigma=d x^{-} d x^{1} d x^{2},
$$


while translations are generated by

$$
P_{-}=\int_{\Sigma} d \sigma T_{-}^{+}=\int_{\Sigma} d \sigma T_{--}, \quad P_{i}=\int_{\Sigma} d \sigma T_{i}^{+}=\int_{\Sigma} d \sigma T_{-i} .
$$

Generators of rotations and boosts may be calculated with Eqs. (55)-(57).

\section{PODOLSKY'S ELECTROMAGNETIC THEORY ON THE NULL-PLANE}

\section{A. Conjugated momenta}

Podolsky's electromagnetic theory is described by the Lagrangian density

$$
L=-\frac{1}{4} F_{\mu \nu} F^{\mu \nu}+\frac{1}{2} a^{2} \partial_{\lambda} F^{\mu \lambda} \partial^{\gamma} F_{\mu \gamma}, \quad F_{\mu \nu} \equiv \partial_{\mu} A_{\nu}-\partial_{\nu} A_{\mu},
$$

in which $A_{\mu}$ are the fundamental fields, components of a $U(1)$ gauge connection. The fields $F_{\mu \nu}$ are components of the curvature field strength related to the connection field. Therefore, density (66) is second-order in the fields $A_{\mu}$ and preserves the $U(1)$ gauge symmetry. In fact, all relativistic second-order Lagrangians with the $U(1)$ symmetry are equivalent to (66). ${ }^{12}$

The action related to (66) is given by

$$
A=\int_{\Omega} d \omega\left(-\frac{1}{4} F_{\mu \nu} F^{\mu \nu}+\frac{1}{2} a^{2} \partial_{\lambda} F^{\mu \lambda} \partial^{\gamma} F_{\mu \gamma}\right),
$$

where $\Omega$ is a 4-volume in Minkowski space-time with 4-volume element $d \omega$. The general first variation of this functional, analog to Eq. (4), is given by

$$
\begin{aligned}
\delta A= & \int_{\Omega} d \omega \frac{\delta L}{\delta A_{\mu}}\left(\delta-\delta x^{\gamma} \partial_{\gamma}\right) A_{\mu} \\
& +\int_{\Omega} d \omega \partial_{\gamma}\left[\frac{\delta L}{\delta\left(\partial_{\gamma} A_{\mu}\right)} \delta A_{\mu}+\frac{\delta L}{\delta\left(\partial_{\gamma} \partial_{\nu} A_{\mu}\right)} \delta\left(\partial_{\nu} A_{\mu}\right)-H_{\mu}^{\gamma} \delta x^{\mu}\right],
\end{aligned}
$$

where

$$
\frac{\delta}{\delta A_{\mu}}=\frac{\partial}{\partial A_{\mu}}-\partial_{\gamma} \frac{\partial}{\partial\left(\partial_{\gamma} A_{\mu}\right)}+\partial_{\gamma} \partial_{\lambda} \frac{\partial}{\partial\left(\partial_{\gamma} \partial_{\lambda} A_{\mu}\right)}
$$

is the Lagrange derivative up to the second-order derivative term, and

$$
H_{\beta}^{\alpha} \equiv \frac{\delta L}{\delta\left(\partial_{\alpha} A_{\mu}\right)} \partial_{\beta} A_{\mu}+\frac{\delta L}{\delta\left(\partial_{\alpha} \partial_{\gamma} A_{\mu}\right)} \partial_{\beta} \partial_{\gamma} A_{\mu}-L \delta_{\beta}^{\alpha}
$$

is the general form of the energy-momentum tensor density.

Observing (70), we have the following covariant momenta:

$$
\begin{gathered}
\pi^{\mu \nu}=\frac{\delta \mathrm{L}}{\delta\left(\partial_{\mu} A_{\nu}\right)}=F^{\mu \nu}-2 a^{2} \eta^{\omega \gamma} \Upsilon_{\beta \omega}^{\alpha \mu} \Delta_{\alpha \gamma}^{\nu \lambda} \partial_{\lambda} \partial_{\rho} F^{\beta \rho}, \\
\pi^{\mu \nu \lambda}=\frac{\delta \mathrm{L}}{\delta\left(\partial_{\mu} \partial_{\nu} A_{\lambda}\right)}=2 a^{2} \eta^{\omega \gamma} \Upsilon_{\beta \omega}^{\alpha \mu} \Delta_{\alpha \gamma}^{\nu \lambda} \partial_{\rho} F^{\beta \rho}
\end{gathered}
$$

where we use the symbols

$$
\Upsilon_{\mu \nu}^{\alpha \beta} \equiv \frac{1}{2}\left(\delta_{\mu}^{\alpha} \delta_{v}^{\beta}-\delta_{\mu}^{\beta} \delta_{v}^{\alpha}\right), \quad \Delta_{\mu \nu}^{\alpha \beta} \equiv \frac{1}{2}\left(\delta_{\mu}^{\alpha} \delta_{v}^{\beta}+\delta_{\mu}^{\beta} \delta_{v}^{\alpha}\right)
$$

In this case, we may also write

$$
H_{\alpha \beta}=F_{\alpha}^{\mu} A_{\mu, \beta}+a^{2} \eta_{\alpha \tau} \Upsilon_{\epsilon \nu}^{\tau \mu} \partial^{\epsilon} \partial_{\lambda} F^{\nu \lambda} A_{\mu, \beta}+2 a^{2} \eta^{\gamma \nu} \eta_{\alpha \phi} \Delta_{\mu \nu}^{\lambda \phi} \Upsilon_{\psi \gamma}^{\mu \epsilon} \partial_{\tau} F^{\psi \tau} A_{\epsilon, \lambda \beta}-\eta_{\alpha \beta} L
$$

as the non-symmetric energy-momentum tensor density. The field equations are written by

$$
\frac{\delta L}{\delta A_{\mu}}=\left[1+a^{2} \square\right] \partial_{\alpha} F^{\mu \alpha}=0 .
$$

In the null-plane dynamics, where the time axis is the unit vector $u^{\alpha}=(1,0,0,0)$ in null-plane coordinates, the Hamiltonian function is given by the expression 


$$
H=\int_{\Sigma} d \sigma H_{\beta}^{\alpha} u_{\alpha} u^{\beta}=\int_{\Sigma} d \sigma H_{+}^{+} .
$$

Again, $\Sigma$ is a 3 -surface of constant $x^{+}$, and $d \sigma \equiv d x^{-} d x^{1} d x^{2}$ is its respective volume element. The Hamiltonian density takes the form

$$
\begin{aligned}
\mathcal{H}_{c} & \equiv H^{+}{ }_{+}=\pi^{\mu+} A_{\mu,+}+\pi^{\mu+v} A_{\mu,+\nu}-L \\
& =\left[\pi^{\mu+}-\partial_{-} \pi^{\mu+-}-\partial_{i} \pi^{\mu+i}\right] A_{\mu,+}+\pi^{\mu++} A_{\mu,++}-L,
\end{aligned}
$$

where $i=1,2$. This Hamiltonian is precisely of the form $\mathcal{H}_{c}=p^{\mu} \bar{A}_{\mu}+\pi^{\mu}\left(\partial_{+} \bar{A}_{\mu}\right)-L$, with

$$
\begin{gathered}
p^{\mu} \equiv \pi^{\mu+}-\partial_{-} \pi^{\mu+-}-\partial_{i} \pi^{\mu+i}, \\
\pi^{\mu} \equiv \pi^{\mu++} .
\end{gathered}
$$

Then, we identify $\left(p^{\mu}, \pi^{\mu}\right)$ as the momenta conjugated to the variables $\left(A_{\mu}, \bar{A}_{\mu}\right)$, respectively. They have the explicit expressions

$$
\begin{gathered}
\pi^{\mu}=a^{2} \eta^{\mu+} \partial_{\rho} F^{+\rho} \\
p^{\mu}=F^{\mu+}+\partial_{+} \pi^{\mu}+2 a^{2}\left(\delta_{\beta}^{\mu} \partial^{+}-\frac{1}{4} \delta_{\beta}^{+} \partial^{\mu}-\frac{1}{2} \eta^{\mu \alpha} \Delta_{\alpha \beta}^{+\lambda} \partial_{\lambda}\right) \partial_{\rho} F^{\beta \rho}
\end{gathered}
$$

\section{B. The HJ equations}

If we write (78) in the null-plane components, we have the following conjugated momenta:

$$
\begin{gathered}
\pi^{+}=0, \\
\pi^{i}=0, \\
\pi^{-}=a^{2} \partial_{\lambda} F^{+\lambda}, \\
p^{+}=\partial_{-} \pi^{-}, \\
p^{-}=F^{-+}+2 a^{2} \partial^{+} \partial_{\lambda} F^{-\lambda}, \\
p^{i}=F^{i+}+2 a^{2} \partial^{+} \partial_{\lambda} F^{i \lambda}-\partial_{i} \pi^{-} .
\end{gathered}
$$

These are the variables conjugated to $\bar{A}_{+}, \bar{A}_{i}, \bar{A}_{-}, A_{+}, A_{-}$, and $A_{i}$, respectively. We observe that the momenta $\pi^{+}, \pi^{i}$, and $p^{+}$are not invertible; thus, we associate them with the canonical constraints

$$
\pi^{+}=0, \quad \pi^{i}=0, \quad p^{+}-\partial_{-} \pi^{-}=0 .
$$

From $p^{-}$, on the other hand, we obtain an expression for $\partial_{+} \bar{A}_{-}$,

$$
\partial_{+} \bar{A}_{-}=\left(2 a^{2} \partial_{-}\right)^{-1}\left[p^{-}-\bar{A}_{-}+\left(1-2 a^{2} \nabla^{2}\right) \partial_{-} A_{+}+2 a^{2} \partial_{-} \partial_{-} \bar{A}_{+}+2 a^{2} \partial_{-} \partial_{i} \bar{A}_{i}\right],
$$

where $\left(2 a^{2} \partial_{-}\right)^{-1}$ represents Green's function of the operator $2 a^{2} \partial_{-}$, which depends on a set of initial/boundary conditions, the characteristic data of the fields. Without specifying these data, (81) is actually a family of solutions. We will need to fix the characteristic data later when analysing the HJ equations of the system, and in fact expression (81) is not required in any of the following analysis. In this case, we let the initial/boundary analysis for later discussion.

Now, the expression for $p^{i}$ can be written in the form

$$
p^{i}=F_{-i}+\partial_{i} \pi^{-}+2 a^{2} \partial_{-}\left(2 \partial_{-} \bar{A}_{i}-\partial_{i} \bar{A}_{-}-\partial_{-} \partial_{i} \bar{A}_{+}+\partial_{j} F_{i j}\right) .
$$

This expression does not provide any velocity; therefore, (80) and (82) constitute the following set of constraints: 


$$
\begin{gathered}
\phi_{1}=\pi^{+}, \\
\phi_{2}^{i}=\pi^{i}, \\
\phi_{3}=p^{+}-\partial_{-} \pi^{-}, \\
\phi_{4}^{i}=p^{i}-\partial_{i} \pi^{-}+F_{i-}+2 a^{2} \partial_{-}\left[\partial_{i} \bar{A}_{-}-2 \partial_{-} \bar{A}_{i}+\partial_{i} \partial_{-} A_{+}-\partial_{j} F_{i j}\right] .
\end{gathered}
$$

Since

$$
\pi^{\mu} \equiv \frac{\partial S}{\partial \bar{A}_{\mu}}, \quad p^{\mu} \equiv \frac{\partial S}{\partial A_{\mu}},
$$

Equation (83) are a set of $\mathrm{HJ}$ equations.

Another HJ equation is necessary, the one related to the canonical Hamiltonian density (76), which has the explicit form

$$
\mathcal{H}_{c}=p^{\mu} \bar{A}_{\mu}+\pi^{-}\left(\partial_{-} \bar{A}_{+}+\partial_{i} \bar{A}_{i}-\nabla^{2} A_{+}\right)-\frac{1}{2} a^{2} \partial_{\lambda} F^{i \lambda} \partial^{\gamma} F_{i \gamma}+\frac{1}{4} F_{\mu \nu} F^{\mu \nu},
$$

where $\nabla^{2}=\partial_{i} \partial_{i}$. Note that this density has no explicit dependency on any velocity, the same happening with the densities in (83). Then, there is no need for (81) in the construction of the constraints. In this case, we simply write the set of $\mathrm{HJ}$ equations

$$
\begin{gathered}
\phi_{0}=p_{0}+\mathcal{H}_{c}, \\
\phi_{1}=\pi^{+}, \\
\phi_{2}^{i}=\pi^{i}, \\
\phi_{3}=p^{+}-\partial_{-} \pi^{-}, \\
\phi_{4}^{i}=p^{i}-\partial_{i} \pi^{-}+F_{i-}+2 a^{2} \partial_{-}\left[\partial_{i} \bar{A}_{-}-2 \partial_{-} \bar{A}_{i}+\partial_{-} \partial_{i} A_{+}-\partial_{j} F_{i j}\right],
\end{gathered}
$$

where $p_{0} \equiv \partial S / \partial x^{+}$is the momentum conjugated to the time variable $x^{+}$. These equations are the final form of the HJ equation (29) for Podolsky's electrodynamics.

\section{Generalised brackets}

To be a complete integrable system, (86) must be a complete involutive set with the complete Poisson brackets,

$$
\begin{aligned}
\{F, G\} \equiv & \int_{\Sigma} d \sigma_{x}\left[\frac{\partial F}{\partial A_{\mu}(x)} \frac{\partial G}{\partial p^{\mu}(x)}-\frac{\partial G}{\partial A_{\mu}(x)} \frac{\partial F}{\partial p^{\mu}(x)}+\right. \\
& \left.+\frac{\partial F}{\partial \bar{A}_{\mu}(x)} \frac{\partial G}{\partial \pi^{\mu}(x)}-\frac{\partial G}{\partial \bar{A}_{\mu}(x)} \frac{\partial F}{\partial \pi^{\mu}(x)}\right]
\end{aligned}
$$

for any observables $F$ and $G$ of the complete phase space of the variables $\left(A_{\mu}, \bar{A}_{\mu}, p^{\mu}, \pi^{\mu}\right)$. Clearly, the fundamental PB is

$$
\left\{A_{\mu}(x), p^{v}(y)\right\}=\left\{\bar{A}_{\mu}(x), \pi^{v}(y)\right\}=\delta_{\mu}^{v} \delta^{3}(x-y),
$$

in which $\delta^{3}(x-y) \equiv \delta\left(x^{-}-y^{-}\right) \delta\left(x^{1}-y^{1}\right) \delta\left(x^{2}-y^{2}\right)$ is the appropriate Dirac's delta.

When calculating the matrix $M \equiv\left\{\phi_{A}, \phi_{B}\right\}$, with $\phi_{A}=\left(\phi_{0}, \phi_{1}, \phi_{2}^{i}, \phi_{3}, \phi_{4}^{i}\right)$, we see that $\phi_{1}$ is in involution, but not the remaining constraints. Particularly, the subset $\left(\phi_{2}^{i}, \phi_{3}, \phi_{4}^{i}\right)$ is non-involutive, but it gives rise to a singular sub-matrix, indicating that a particular linear combination of these constraints is integrable. However, we have the subset $\left(\phi_{2}^{i}, \phi_{4}^{i}\right)$, which obeys the relations

$$
\begin{gathered}
\left\{\phi_{2}^{i}(x), \phi_{4}^{j}(y)\right\}=-4 a^{2} \eta^{i j} \partial_{-}^{x} \partial_{-}^{x} \delta^{3}(x-y), \\
\left\{\phi_{4}^{i}(x), \phi_{4}^{j}(y)\right\}=2 \eta^{i j}\left[1-2 a^{2} \nabla_{x}^{2}\right] \partial_{-}^{x} \delta^{3}(x-y) .
\end{gathered}
$$


This particular subset of $\mathrm{HJ}$ equations gives rise to the matrix

$$
M_{I J}^{i j} \equiv 2 \eta^{i j}\left(\begin{array}{cc}
0 & -2 a^{2} \partial_{-}^{x} \partial_{-}^{x} \\
2 a^{2} \partial_{-}^{x} \partial_{-}^{x} & \partial_{-}^{x}\left[1-2 a^{2} \nabla_{x}^{2}\right]
\end{array}\right) \delta^{3}(x-y), \quad I, J=2,4 .
$$

A matrix $\left(M^{-1}\right)_{i j}^{I J}$ obeying

$$
\int d^{3} z M_{I J}^{i j}(x, z)\left(M^{-1}\right)_{j k}^{J K}(z, y)=\int d^{3} z\left(M^{-1}\right)_{j k}^{J K}(x, z) M_{I J}^{i j}(z, y)=\delta_{k}^{i} \delta_{I}^{K} \delta^{3}(x-y),
$$

if unique, is defined as the inverse matrix of $M_{I J}^{i j}$. A solution for (91) is given by

$$
\left(M^{-1}\right)_{i j}^{I J}(x, y) \equiv \frac{1}{2} \eta_{i j}\left(\begin{array}{cc}
\alpha(x, y) & \beta(x, y) \\
-\beta(x, y) & 0
\end{array}\right)
$$

with

$$
\begin{gathered}
\alpha(x, y)=\frac{1}{4 a^{2}}\left|x^{-}-y^{-}\right|^{2} \epsilon\left(x^{-}-y^{-}\right)\left[1-2 a^{2} \nabla_{x}^{2}\right] \delta^{2}(\mathbf{x}-\mathbf{y}), \\
\beta(x, y)=\frac{1}{a^{2}}\left|x^{-}-y^{-}\right| \delta^{2}(\mathbf{x}-\mathbf{y}) .
\end{gathered}
$$

In (93) we have $\delta^{2}(\mathbf{x}-\mathbf{y}) \equiv \delta\left(x^{1}-y^{1}\right) \delta\left(x^{2}-y^{2}\right)$ and the sign function

$$
\epsilon(x-y) \equiv \begin{cases}1, & x>y \\ 0, & x=y \\ -1, & x<y\end{cases}
$$

To each of the functions (93), we may add arbitrary functions of $x^{+}, x^{1}$, and $x^{2}$ and polynomials of $x^{-}$up to second-order. Therefore, we do not have a unique inverse. These functions can be fixed using proper boundary conditions for the fields in a null-plane of constant $x^{-}$. We choose these conditions to be $\partial_{-} A_{\mu}=\partial_{-}^{2} A_{\mu}=\partial_{-}^{3} A_{\mu}=0$ for $x^{-} \rightarrow-\infty$. In this case, we may treat (92) as an appropriate inverse matrix.

The GBs related to the subset $\left(\phi_{2}^{i}, \phi_{4}^{i}\right)$ are given by

$$
\{F, G\}^{*} \equiv\{F, G\}-\iint_{\Sigma} d \sigma_{x} d \sigma_{y}\left\{F, \phi_{I}^{i}(x)\right\}\left(M^{-1}\right)_{i j}^{I J}(x, y)\left\{\phi_{J}^{j}(y), G\right\}
$$

and result in the fundamental relations

$$
\begin{aligned}
\left\{A_{\mu}(x), \bar{A}_{v}(y)\right\}^{*}= & -\frac{1}{2} \eta_{\mu j} \delta_{v}^{j} \beta(x, y), \\
\left\{A_{\mu}(x), p^{v}(y)\right\}^{*}= & \delta_{\mu}^{v} \delta^{3}(x-y), \\
\left\{\bar{A}_{\mu}(x), \bar{A}_{v}(y)\right\}^{*}= & \frac{1}{2} \eta_{\mu j} \delta_{v}^{j} \alpha(x, y)+\Delta_{\mu \nu}^{j-} \partial_{j}^{x} \beta(x, y), \\
\left\{\bar{A}_{\mu}(x), p^{v}(y)\right\}^{*}= & \frac{1}{2} \delta_{\mu}^{j} \delta_{-}^{v} \partial_{i}^{x} \beta(x, y) \\
& -a^{2} \delta_{\mu}^{i}\left[\delta_{k}^{v} \partial_{k}^{x} \partial_{i}^{x}-\delta_{+}^{v} \partial_{i}^{x} \partial_{-}^{x}+\delta_{i}^{v}\left(\frac{1}{2 a^{2}}-\nabla_{x}^{2}\right)\right] \partial_{-}^{x} \beta(x, y), \\
\left\{\bar{A}_{\mu}(x), \pi^{v}(y)\right\}^{*}= & \delta_{\mu}^{v} \delta^{3}(x-y)+a^{2} \delta_{\mu}^{i}\left[\delta_{-}^{v} \partial_{i}^{x}-2 \delta_{i}^{v} \partial_{-}^{x}\right] \partial_{-}^{x} \beta(x, y) .
\end{aligned}
$$

Since the subset $\left(\phi_{2}^{i}, \phi_{4}^{i}\right)$ is eliminated, we have $\left\{\phi_{1}, \phi_{3}\right\}^{*}=0$ and $\left\{\phi_{0}, \phi_{1}\right\}^{*}=\phi_{3}$. However,

$$
\left\{\phi_{0}(x), \phi_{3}(y)\right\}^{*}=-\left[\partial_{-}^{x} p^{-}(x)+\partial_{i}^{x} p^{i}(x)\right] \delta^{3}(x-y) .
$$


Equation (97) implies a new HJ equation, named

$$
\Phi(x) \equiv-\partial_{-}^{x} p^{-}(x)-\partial_{i}^{x} p^{i}(x)=0 .
$$

Now we have the extended and renamed set of constraints

$$
\begin{gathered}
\Phi_{0} \equiv p_{0}+\mathcal{H}_{c}, \\
\Phi_{1} \equiv \pi^{+}(x), \\
\Phi_{2} \equiv p^{+}(x)-\partial_{-}^{x} \pi^{-}(x), \\
\Phi_{3} \equiv-\partial_{-}^{x} p^{-}(x)-\partial_{i}^{x} p^{i}(x) .
\end{gathered}
$$

Calculating the GB between them, we see that this set is complete and in involution with the GB operation. Therefore, we have completed the task at hand, which was to find a complete integrable set of $\mathrm{HJ}$ equations for the system.

\section{The field equations}

With GB (95), the fundamental differential

$$
d F(x)=\int_{\Sigma} d \sigma_{y}\left\{F(x), \Phi_{A}(y)\right\}^{*} d t^{A}(y), \quad A=(0,1,2,3),
$$

gives the dynamical evolution of any observable $F(A, \bar{A}, p, \pi)$ of the phase space of the system. The set of HJ equations (99), represented here by the set $\Phi_{A}(A, \bar{A}, p, \pi)=0$, is completely integrable due to the analysis made in Sec. IV C and provides a set of dynamical generators for the evolution of the system. They may also be called Hamiltonians of the system. Each Hamiltonian $\Phi_{A}$ is related to an independent variable (or parameter) $t^{A}$, which is completely arbitrary in principle. We know, of course, that some of the parameters must be related to some of the phase space variables, and, particularly, $t^{0}=x^{+}$is found when $F=x^{+}$in (100). The parameter $t^{3}$, on the other hand, is not a part of the original set of independent variables since $\Phi_{3}$ is a generator found later in the integrability analysis, and no phase space variable can be related to it, in principle. Nevertheless, the parameter space is expanded to contain as many parameters as the number of involutive constraints of the system as long as integrability is assured. Moreover, the Frobenius theorem implies linear independence of the independent variables, so the evolution in a given "direction" does not depend on other "directions" of the parameter space.

For $A_{\mu}$, we have the characteristic equation

$$
d A_{\mu}=\bar{A}_{\mu} d t^{0}+\delta_{\mu}^{+} d t^{2}+\left(\delta_{\mu}^{-} \partial_{-}+\delta_{\mu}^{i} \partial_{i}\right) d t^{3} .
$$

Since $t^{0}=x^{+}$, time evolution is given by

$$
\partial_{+} A_{+}=\bar{A}_{+}, \quad \partial_{+} A_{-}=\bar{A}_{-}, \quad \partial_{+} A_{i}=\bar{A}_{i},
$$

as expected. The characteristic equation associated with $\bar{A}_{\mu}$ is

$$
d \bar{A}_{\mu}=\delta_{\mu}^{-}\left[\partial_{-} \bar{A}_{+}+\partial_{i} \bar{A}_{i}-\nabla^{2} A_{+}\right] d t^{0}+\delta_{\mu}^{+} d t^{1}+\left[\delta_{\mu}^{-} \partial_{-}+\delta_{\mu}^{i} \partial_{i}\right] d t^{2} .
$$

Time evolution alone yields

$$
\partial_{+} \bar{A}_{+}=0, \quad \partial_{+} \bar{A}_{i}=0, \quad \partial_{+} \bar{A}_{-}=\partial_{-} \bar{A}_{+}+\partial_{i} \bar{A}_{i}-\nabla^{2} A_{+},
$$

which are expected. Particularly, the $\partial_{+} \bar{A}_{-}$equation agrees with (81) when the constraints are used.

The characteristic equations associated with the canonical momenta $p^{\mu}$ and $\pi^{\mu}$ are obtained from (100), resulting in the expressions

$$
\begin{gathered}
\partial_{+} p^{+}=\partial_{\lambda} F^{\lambda+}-a^{2} \partial_{i} \partial_{-} \partial_{\lambda} F^{\lambda i}-a^{2} \nabla^{2} \partial_{\lambda} F^{\lambda+}, \\
\partial_{+} p^{i}=\partial_{-} F^{-i}+\left(1-a^{2} \square\right) \partial_{j} F^{i j} \\
\partial_{+} p^{-}=\partial_{i} F^{i-} .
\end{gathered}
$$


Similarly, for $\pi^{\mu}$, we derive

$$
\begin{gathered}
\partial_{+} \pi^{+}=\phi_{3}, \\
\partial_{+} \pi^{+}=F_{+-}-p^{-}+a^{2} \partial_{i} \partial_{\lambda} F^{\lambda i}, \\
\partial_{+} \pi^{i}=-\phi_{4}^{i} .
\end{gathered}
$$

The relations associated with $\pi^{+}$and $\pi^{-}$indicate what we saw from the integrability conditions, i.e., $\phi_{3}$ and $\phi_{4}^{i}$ result from the conditions $d \phi_{1}=0$ and $d \phi_{2}^{i}=0$. Using the fact that $\partial_{+} \pi^{+}=0, \partial_{+} \pi^{i}=0$, and $\pi^{-}=a^{2} \partial_{\lambda} F^{+\lambda}$, we obtain

$$
\begin{gathered}
p^{+}=a^{2} \partial_{-} \partial_{\lambda} F^{+\lambda}, \\
p^{-}=F^{-+}+a^{2} \partial_{\lambda} F^{-\lambda}, \\
p^{i}=F^{+i}-a^{2} \partial_{i} \partial_{+} \partial_{\lambda} F^{\lambda+}-2 a^{2} \partial_{-} \partial_{+} \partial_{\lambda} F^{\lambda i} .
\end{gathered}
$$

Substituting (107) in (106), we deduce the field equations

$$
\left(1+a^{2} \square\right) \partial_{\lambda} F^{\mu \lambda}=0
$$

Therefore, the time evolution sector of the characteristic equations is equivalent to the Lagrangian field equations.

\section{E. Symmetries}

Let us go back to the complete set of dynamical generators (99) with the canonical Hamiltonian density (85). The Hamiltonians $\Phi_{A}$, as shown in Ref. 7, are generators of canonical transformations in the complete phase space. The $x^{+}$"time" evolution studied above is a particular case. As usual in the constraint analysis, we are also interested in the canonical transformations that represent Lagrangian gauge transformations. In this case, we consider the following infinitesimal transformations:

$$
\delta F(x)=\int_{\Sigma} d \sigma_{y}\left\{F(x), \Phi_{a}(y)\right\}^{*} \delta \omega^{a}(y), \quad a=(1,2,3),
$$

which are taken with $\delta x^{+}=0$. The generator is given by

$$
G \equiv \int_{\Sigma} d \sigma_{x} \Phi_{a}(x) \delta \omega^{a}(x), \quad a=1,2,3,
$$

with $\Phi_{a}=0$.

Now we calculate a fixed point transformation on the Lagrangian density (66), given by

$$
\delta L=\delta A_{\mu} \frac{\delta L}{\delta A_{\mu}}=\delta A_{\mu}\left(\square+m^{2}\right) \partial_{\alpha} F^{\mu \alpha}, \quad m^{2} \equiv 1 / a^{2},
$$

apart from a total divergence. Remember that $\delta L / \delta A_{\mu}$ is the Lagrange derivative (69). On the other hand, $\delta A_{\mu}$ is generated by $G$ via the generalised brackets, which gives the result

$$
\delta A_{\mu}=\left\{A_{\mu}, G\right\}^{*}=\delta_{\mu}^{+} \delta \omega^{2}-\left(\delta_{\mu}^{-} \partial_{-}+\delta_{\mu}^{i} \partial_{i}\right) \delta \omega^{3} .
$$

Apart from divergence terms, substitution of (112) in (111) yields

$$
\delta L=\delta \omega^{2}\left(\square+m^{2}\right) \partial_{\alpha} F^{+\alpha}-\delta \omega^{3}\left(\square+m^{2}\right)\left(\partial_{-} \partial_{\alpha} F^{-\alpha}+\partial_{i} \partial_{\alpha} F^{i \alpha}\right) .
$$

Note that the identity $\partial_{\alpha} \partial_{\beta} F^{\alpha \beta}=0$ results in

$$
\partial_{-} \partial_{\alpha} F^{-\alpha}+\partial_{i} \partial_{\alpha} F^{i \alpha}=-\partial_{+} \partial_{\alpha} F^{+\alpha},
$$

so, after some calculation,

$$
\delta L=\left(\delta \omega^{2}+\partial_{+} \delta \omega^{3}\right)\left(\square+m^{2}\right) \partial_{\alpha} F^{+\alpha} .
$$

The system is gauge invariant under transformation (112) if $\delta \mathcal{L}$ is zero in $\Omega$. Of course, the field equations themselves lead to $\delta \mathcal{L}=0$, but if the symmetry is understood to be valid outside the solutions of the variational problem, we must have

$$
\delta \omega^{2}=-\partial_{+} \delta \omega^{3} .
$$


Supposing $\Lambda \equiv \delta \omega^{3}$ the gauge parameter, we have

$$
\delta \omega^{2}=-\partial_{+} \Lambda
$$

so (112) gives

$$
\delta A_{\mu}=-\delta_{\mu}^{+} \partial_{+} \Lambda-\delta_{\mu}^{-} \partial_{-} \Lambda-\delta_{\mu}^{i} \partial_{i} \Lambda=-\partial_{\mu} \Lambda .
$$

Therefore, we recover the correct gauge transformations $A_{\mu} \rightarrow A_{\mu}-\partial_{\mu} \Lambda$.

On the other hand, taking $\partial_{+} \Lambda \equiv \dot{\Lambda}$,

$$
\delta \bar{A}_{\mu}=\delta_{\mu}^{+} \delta \omega^{1}-\delta_{\mu}^{-} \partial_{-} \dot{\Lambda}-\delta_{\mu}^{i} \partial_{i} \dot{\Lambda},
$$

and $\delta \omega^{1}$ is still arbitrary. However, if the gauge transformation for $A_{\mu}$ is given by $\delta A_{\mu}=-\partial_{\mu} \Lambda$, the same transformation for $\bar{A}_{\mu}$ should be $\delta \bar{A}_{\mu}=-\partial_{\mu} \dot{\Lambda}$ for the sake of consistency. Therefore, we should fix $\delta \omega^{1}=-\ddot{\Lambda}$, so

$$
\delta \bar{A}_{\mu}=-\delta_{\mu}^{+} \ddot{\Lambda}-\delta_{\mu}^{-} \partial_{-} \dot{\Lambda}-\delta_{\mu}^{i} \partial_{i} \dot{\Lambda}=-\partial_{\mu} \dot{\Lambda} .
$$

After some calculation, the generator of gauge transformations (110) becomes

$$
G=\int_{\Sigma} d \sigma_{x}\left(\partial_{\mu} \pi^{\mu} \dot{\Lambda}+\partial_{\mu} p^{\mu} \Lambda\right) .
$$

In fact, generator (118) reproduces relations (116) and (117).

\section{FINAL REMARKS}

In this paper, we analysed the null-plane canonical structure of Podolsky's generalised electrodynamics via the Hamilton-Jacobi formalism. The essence of the HJ approach is to understand canonical constraints as generators of transformations on the phase space. Each generator is a Hamiltonian function responsible for the evolution of the system along a respective evolution parameter as the canonical Hamiltonian function which is the generator of time evolution. The flows generated by the Hamiltonians are solutions of the characteristic equations taken from the fundamental differential (47). If complete integrability is assured by the application of Frobenius' conditions, these generators are in involution with the generalised brackets, closing themselves a Lie algebra. A complete set of involutive constraints generates transformations that preserve the symplectic structure of the phase space, therefore becoming canonical transformations, whose flows are called characteristic flows. In this case, we understand the HJ formalism as the search for a complete set of involutive Hamiltonians of a singular system.

Applied to Podolsky's theory, the HJ approach starts with the definition of constraints (86). Among these constraints, we identify two sets of non-involutive ones, leading to GB (95), with fundamental relations calculated in (96). These brackets are defined only with the application of proper boundary conditions, $\partial_{-} A_{\mu}=\partial_{-}^{2} A_{\mu}=\partial_{-}^{3} A_{\mu}=0$ for $x^{-} \rightarrow-\infty$. The GB eliminates the set $\left(\phi_{2}^{i}, \phi_{4}^{i}\right)$ but reveals a new constraint given by (98). The complete set of Hamiltonians, written in (99), is in involution with the GB, therefore completing the task of finding the complete set of generators of the system.

In Sec. IV D, the canonical field equations are calculated. It is shown that these characteristic equations, in the temporal sector, are equal to the Euler-Lagrange equations of Podolsky's Lagrangian.

On the other hand, the evolution of the system in the direction of the remaining independent variables $\omega^{a}$, with $\delta x^{+}=0$, is analysed in Sec. IV E. In this case, the Hamiltonians $\Phi_{a}$ generate characteristic flows defined by (110), and these flows are symmetries of the system. Since $\Phi_{a}$ form a complete set of compatible observables in the reduced phase space, we expect that these transformations are related to the gauge transformations defined by an invariant field strength $F_{\mu \nu}$. In fact, $\delta L=0$ leads directly to relation (114) between the former independent variables. Choosing $\Lambda \equiv \delta \omega^{3}$ as the gauge parameter, we see that $\omega^{2}$ must have the form $\delta \omega^{2}=-\dot{\Lambda}$, while $\omega^{1}$ must obey $\delta \omega^{1}=-\ddot{\Lambda}$. The correct gauge transformations (116) and (117) are generated by generating function (118).

Here, we stress the fact that the application of the HJ theory not only provides straightforward results for the complete set of generators and consistent canonical field equations but also results in the correct generators of the gauge transformations via the correct relation among the independent 
variables of the theory. This last result was always of some controversy in the literature since Dirac's method incorporates an unproven assumption, Dirac's conjecture, which is the statement that all firstclass constraints (corresponding to our involutive constraints) must contribute to the construction of the generator. Moreover, no ad hoc method, as Castellani's procedure, ${ }^{28}$ was necessary. In fact, it seems that Dirac's conjecture is actually not a defined problem in the HJ formalism. It is our intent, however, to study the relation between Frobenius' theorem and this conjecture in the near future.

The HJ formalism can also be applied to other interesting models with higher-order Lagrangians as the already mentioned new massive gravity ${ }^{10}$ and generalised massive gravity ${ }^{11}$ in three and four spacetime dimensions. It is possible that an alternative approach to these systems can shed light upon open problems in 3D gravity, e.g., new examples and applications of the holographic correspondence. The authors are also investigating the non-abelian Podolsky theory. The aim is to understand how, in the $\mathrm{HJ}$ approach, higher derivatives could address problems like gauge freedom in generalised gauge theories.

\section{ACKNOWLEDGMENTS}

M.C.B. thanks CAPES and FAPESP for partial support and B.M.P. thanks CNPq for partial support. C.E.V. was supported by CNPq process No. 150407/2016-5 and G.E.R.G. was supported by VIPRI-UDENAR.

${ }^{1}$ C. Carathéodory, Calculus of Variations and Partial Differential Equations of the First Order (AMS Chelsea Publishing, 2000).

${ }^{2} \mathrm{H}$. Rund, The Hamilton-Jacobi Theory in the Calculus of Variations; Its Role in Mathematics and Physics (Van Nostrand, 1966).

${ }^{3}$ P. A. M. Dirac, "Generalized Hamiltonian dynamics," Can. J. Math. 2, 129 (1950); “The Hamiltonian form of field dynamics," 3, 1 (1951); Lectures on Quantum Mechanics (Yeshiva University, New York, 1964).

${ }^{4}$ J. L. Anderson and P. G. Bergmann, "Constraints in covariant field theories," Phys. Rev. 83, 1018 (1951); P. G. Bergmann and I. Goldberg, "Dirac bracket transformations in phase space," ibid. 98, 531 (1955).

${ }^{5}$ Y. Güler, "Integration of singular systems," Il Nuovo Cimento B 107, 1143 (1992); Canonical formulation of singular systems," 107, 1389 (1992).

${ }^{6}$ M. C. Bertin, B. M. Pimentel, and C. E. Valcárcel, "Non-involutive constrained systems and Hamilton-Jacobi formalism," Ann. Phys. 323, 3137 (2008).

${ }^{7}$ M. C. Bertin, B. M. Pimentel, and C. E. Valcárcel, "Involutive constrained systems and Hamilton-Jacobi formalism,” J. Math. Phys. 55, 112901 (2014).

${ }^{8}$ K. S. Stelle, "Renormalization of higher-derivative quantum gravity," Phys. Rev. D 16, 953 (1977).

${ }^{9}$ K. S. Stelle, "Classical gravity with higher derivatives," Gen. Relativ. Gravitation 9, 353 (1978); L. Querella, "Variational principles and cosmological models in higher-order gravity," Ph.D thesis, e-print arXiv: gr-qc/9902044, and references therein.

${ }^{10}$ E. A. Bergshoe, O. Hohm, and P. K. Townsend, "Massive gravity in three dimensions," Phys. Rev. Lett. 102, 201301 (2009); More on massive 3D gravity," Phys. Rev. D 79, 124042 (2009).

${ }^{11}$ M. C. Bertin, D. Grumiller, D. Vassilevich, and T. Zojer, "Generalised massive gravity one-loop partition function and AdS/(L)CFT," J. High Energy Phys. 2011, 111; M. C. Bertin, S. Ertl, H. Ghorbani, D. Grumiller, N. Johansson, and D. Vassilevich, "Lobachevsky holography in conformal Chern-Simons gravity," ibid. 2013, 15; M. Riegler, "How general is holography?," Ph.D thesis, e-print arXiv: 1609.02733 (2016).

${ }^{12}$ R. R. Cuzinatto, C. A. M. de Melo, and P. J. Pompeia, "Second order gauge theory," Ann. Phys. 322, 1211 (2007); R. R. Cuzinatto, C. A. M. de Melo, L. G. Medeiros, and P. J. Pompeia, "Gauge formulation for higher order gravity," Eur. Phys. J. C 53, 99 (2008).

${ }^{13}$ A. I. Alekseev, B. A. Arbozov, and V. A. Baikov, "Infrared asymptotic behavior of gluon Green's functions in quantum chromodynamics," Theor. Math. Phys. 52, 739 (1982).

${ }^{14}$ B. M. Pimentel and R. G. Teixeira, "Hamilton-Jacobi formulation for singular systems with second order lagrangians," Il Nuovo Cimento B 111, 841 (1996); Generalization of the Hamilton-Jacobi approach for higher order singular systems," 113, 805 (1998); M. C. Bertin, B. M. Pimentel, and P. J. Pompeia, "Hamilton-Jacobi approach for first order actions and theories with higher derivatives," Ann. Phys. 323, 527 (2008).

${ }^{15}$ M. Ostrogradsky, Mem. Ac. St. Petersbourg 1, 385 (1850).

${ }^{16}$ D. S. Kaparulin, S. L. Lyakhovich, and A. A. Sharapov, "Classical and quantum stability of higher-derivative dynamics," Eur. Phys. J. C 74, 3072 (2014).

${ }^{17}$ R. P. Woodard, "The theorem of Ostrogradsky," e-print arXiv:1506.02210 (2015).

${ }^{18}$ F. Bopp, "Eine lineare theorie des elektrons," Ann. Phys. 430, 345 (1940).

${ }^{19}$ B. Podolsky and P. Schwed, "Review of a generalized electrodynamics," Rev. Mod. Phys. 20, 40 (1948).

${ }^{20}$ J. Frenkel, “4/3 problem in classical electrodynamics," Phys. Rev. E 54, 5859 (1996).

${ }^{21}$ E. R. Williams, J. E. Faller, and H. A. Hill, "New experimental test of Coulomb's law: A laboratory upper limit on the photon rest mass," Phys. Rev. Lett. 26, 721 (1971); L. Davis, A. S. Goldhaber, and M. M. Nieto, "Limit on the photon mass deduced from Pioneer-10 observations of Jupiter's magnetic field," Phys. Rev. Lett. 35, 1402 (1975). 
22 J. Luo, L.-C. Tu, Z.-K. Hu, and En-J. Luan, "New experimental limit on the photon rest mass with a rotating Torsion balance," Phys. Rev. Lett. 90, 081801 (2003).

${ }^{23}$ P. A. M. Dirac, "Forms of relativistic dynamics," Rev. Mod. Phys. 21, 392 (1949); B. L. G. Bakker, Forms of Relativistic Dynamics, Lecture Notes in Physics (Springer, New York, 2001), Vol. 572.

${ }^{24}$ M. Huszár, "Light front quantization by Dirac's method," J. Phys. A: Math. Gen. 9, 1359 (1976).

${ }^{25}$ P. J. Steinhardt, "Problems of quantization in the infinite momentum frame," Ann. Phys. 128, 425 (1980).

${ }^{26}$ C. A. P. Galvão and B. M. Pimentel, "The canonical structure of Podolsky generalized electrodynamics," Can. J. Phys. 66 460-466 (1988).

${ }^{27}$ M. C. Bertin, B. M. Pimentel, and G. E. R. Zambrano, "The canonical structure of Podolsky's generalized electrodynamics on the null-plane," J. Math. Phys. 52, 102902 (2011).

${ }^{28}$ L. Castellani, "Symmetries in constrained Hamiltonian systems," Ann. Phys. 143, 357 (1982).

${ }^{29} \mathrm{H}$. Weyl, "Geodesic fields in the calculus of variation for multiple integrals," Ann. Math. 36, 607 (1935).

${ }^{30}$ R. R. Cuzinatto, C. A. M. De Melo, L. G. Medeiros, and P. J. Pompeia, "How can one probe Prodolsky electrodynamics?," Int. J. Mod. Phys. A 26, 3641 (2011). 